\title{
Labyrinthe
}

18 | 2004 (2)

La Recherche dans tous ses éclats

\section{Les soldats de Salamine}

De la fabrique de l'histoire à la fabrique de la fiction, une impossible épopée

\section{Héloïse Hermant}

\section{(2) OpenEdition}

\section{Journals}

Édition électronique

URL : http://journals.openedition.org/labyrinthe/226

DOI : $10.4000 /$ labyrinthe. 226

ISSN : 1950-6031

Éditeur

Hermann

Édition imprimée

Date de publication : 15 juillet 2004

Pagination : 89-103

Référence électronique

Héloïse Hermant, "Les soldats de Salamine », Labyrinthe [En ligne], 18 | 2004 (2), mis en ligne le 24 juin 2008, consulté le 19 avril 2019. URL : http://journals.openedition.org/labyrinthe/226 ; DOI : 10.4000/ labyrinthe.226

Propriété intellectuelle 


\title{
LES SOLDATS DE SALAMINE De la fabrique de l'histoire à la fabrique de la fiction, une impossible épopée
}

\author{
Héloïse HERMANT \\ Heloise.Hermant@wanadoo.fr
}

Le roman de Javier Cercas, Les Soldats de Salamine ${ }^{1}$, constitue un étrange objet. En résumer l'intrigue et dérouler le complexe écheveau du récit n'épuise en rien les interrogations qu'il suscite. Peut-on se contenter d'y voir l'antidestin de Sanchez Mazas, écrivain et idéologue cofondateur de la Phalange? N'y a-t-il vraiment que l'histoire de cet homme doublement miraculé, qui échappe à la dernière exécution de soldats fascistes organisée au sanctuaire du Collell par les républicains en déroute et qui, découvert peu après par un milicien, est épargné par ce dernier au terme d'un intense échange de regards? Ce serait déjà beaucoup. Mais c'est plus encore. Que répondre à l'énigme que renferme le titre du roman, Les Soldats de Salamine? Énigme pour le lecteur peu familier d'histoire antique. Mais énigme surtout pour celuilà même qui connaîtrait cet épisode des guerres médiques, et saurait mesurer la symbolique de cette défaite navale perse, en 480 avant JésusChrist: cristallisation de la douleur des vaincus qui voient la gloire de leur patrie s'abîmer dans un désastre irrémédiable, tandis qu'émerge la puissance athénienne.

Qui sont les soldats de Salamine de Cercas ? La lecture du roman ne dissipe pas le mystère, qui est aussi celui des fondements d'une guerre fratricide, de toutes les guerres fratricides, même si l'on pressent que l'essentiel se cache dans le silence des regards échangés par un fasciste traqué et un républicain au bord de la défaite. Le paradoxe consiste en ce qu'il n'y a plus ni vaincu ni vainqueur: le clivage simpliste mais efficace des grandes gestes d'antan n'opère plus. Dans ces conditions, l'allusion à la défaite perse n'est-elle qu'une métaphore 
creuse? Sûrement pas, ne serait-ce qu'en raison du jeu d'échos qui s'instaure entre le titre même du roman et sa structure: une lecture attentive apprend qu'il s'agit du titre du livre que Mazas lui-même projetait d'écrire.

Au moment où le lecteur comprend qu'une analyse minutieuse de l'agencement de la diégèse constitue un préalable indispensable à l'élucidation de l'énigme, il est déjà happé par le récit. Divisé en trois actes aux titres poétiques et sibyllins, «Les amis de la forêt », " Les soldats de Salamine » et «Rendez-vous à Stockton», le roman de Cercas entremêle les strates textuelles. L'écrivain commence par exposer la genèse d'un récit, Les Soldats de Salamine, qui constitue lui-même la deuxième partie du roman, et s'organise autour de l'épisode de l'exécution manquée de Mazas, de sa confrontation muette avec le soldat républicain qui l'épargne, de sa fuite et de ses conséquences. Mais c'est dans la dernière séquence que le romancier «rencontre» véritablement l'authentique héritier des soldats de Salamine, seul héros digne de ce nom, en la personne de Miralles que tout porte à identifier comme le milicien ayant épargné le poète phalangiste. C'est pourquoi le récit des «Soldats de Salamine» auquel fait écho le titre du roman dans sa totalité, n'a rien d'un objet fini enchâssé entre une genèse - voire une gestation - et un épilogue. Il ne s'achève qu'au terme de la troisième partie bâtie sur la version républicaine de la scène de l'échange de regards. Et pourtant ce récit reste virtuel. D'abord, parce que Miralles ne lève pas le doute qui plane sur son identité. Ensuite, parce que Cercas préfère retranscrire sa rencontre avec le vétéran catalan et leurs échanges à bâtons rompus, sans reconstituer la totalité du puzzle. Le récit «idéal» des Soldats de Salamine apparaît sous une forme inachevée. Mais pouvait-il en être autrement?

En somme, le titre énigmatique du roman de Cercas invite à démêler l'écheveau complexe des rapports entre histoire, roman, fiction, mémoire et légende. Parce que la fiction relève plus d'une dynamique textuelle que d'un genre aux critères immuables, l'auteur jubile en se mouvant dans un indéfini où la frontière entre vérité et fiction est en perpétuel mouvement. Il s'amuse à multiplier les apories qu'entraînent les oscillations d'un registre à l'autre. Ainsi, dans Les Soldats de Salamine, plusieurs problèmes liés à cette thématique s'agrègent sans trouver de solution. Mais, après tout, n'est-ce pas le propre d'une énigme que de n'avoir pas de réponse définitive? 


\section{Les soldats de Salamine}

\section{Le dédale de la structure narrative}

Une analyse de la construction labyrinthique du récit permet d'appréhender l'habileté de l'auteur à brouiller les frontières entre histoire et fiction. L'intrigue centrale se détache sans équivoque: il s'agit d'une enquête, journalistique et historique, sur le phalangiste Mazas, son exécution manquée au Collell et sa confrontation muette, le temps d'un jeu de regards, avec un frère ennemi qui l'épargne. Le récit naît et se déploie en même temps que le personnage ressuscite. Mazas n'est d'abord qu'un nom évanescent, puis Cercas, tenu de rédiger un article commémoratif sur la guerre civile, est mis en contact avec un ancien ami du phalangiste. Lentement, la figure fantomatique prend vie tandis que s'amorce la quête vers l'élucidation du mystère entourant le poète: de fil en aiguille, le journaliste interroge des historiens de la guerre civile, des gens qui ont côtoyé Mazas, dévore les archives et rencontre les «amis de la forêt», ces compagnons d'infortune du phalangiste en cavale, républicains déserteurs dans l'attente de l'arrivée des nationalistes vainqueurs. Après la phase d'accumulation de témoignages, marquée par un glissement des sources de seconde main à une confrontation directe avec les acteurs du drame, vient l'heure du récit complet de l'épisode du Collell et de ses suites «romanesques» bien qu'attestées. Vient l'heure où Mazas semble resurgir d'entre les morts, s'emparer de la plume du scripteur pour écrire le récit qu'il avait promis à ses «amis de la forêt» en leur faisant ses adieux en 1939: Les Soldats de Salamine. Car le style indirect libre, les notations poétiques, les plongées dans le for intérieur du phalangiste annulent la distance entre ce dernier et Cercas. Enfin, dans le diminuendo du dernier acte, Mazas s'efface devant l'irruption inattendue de Miralles. C'est alors seulement que l'ensemble du triptyque devient Les Soldats de Salamine de Cercas et non plus ceux de Mazas.

Pourtant, au-delà de cette ligne harmonique simple, des coupes verticales révèlent le feuilletage de la structure narrative où viennent se chevaucher et s'imbriquer plusieurs niveaux de réalité. S'enclenche alors l'oscillation hypnotique de l'histoire à la fiction et de la vérité au mensonge. Si l'on part du cœur du récit en allant du plus «fictionnel» au plus «réel», on repère des niveaux de réalité différents. D'un côté, le «réel» où se meut le personnage principal, et d'un autre, le récit qui est le traitement de la matière brute patiemment amassée. L'oscillation entre ces deux pôles recoupe la dimension auto-réflexive du récit, sa 
mise en abyme. Quand Cercas quitte le noyau dur de la fiction, il est obligé de multiplier les renvois à une réalité extra-textuelle. Ainsi la figure historique de Mazas, les méandres de sa vie minutieusement reconstitués, puis l'Espagne de l'époque, déchirée par la guerre civile, dont le romancier présente une véritable radiographie à l'appui d'un tissu événementiel dense nourri par les travaux des historiens de la période; replacé dans son temps, le poète phalangiste devient, au-delà de sa singularité, le reflet d'une Espagne qui a vu toute une génération basculer dans le fascisme ou le communisme. Ou encore, non sans une touche d'humour, Cercas qui se met lui-même en scène sous son propre nom en tant que narrateur des Soldats de Salamine. Mais il arrive que les références à un au-delà du texte soient partiellement démenties: bien que le narrateur porte le même nom que le romancier, il n'y a pas de correspondances systématiques entre les deux; le «vrai» Cercas n'est pas journaliste et, comme il l'atteste lui-même, il ne partage pas les conceptions de son héros homonyme sur la théorie du roman. Nouveau brouillage des pistes et comble de la perplexité pour le lecteur. Perplexité qu'entretient la nature du pacte implicite entre le lecteur et le scripteur: la structure originale, découpée en titres métaphoriques qui ne livrent pas d'emblée leur contenu, invite à une lecture active misant sur le plaisir de la reconstruction. Or, ce type d'implication est celui d'un lecteur de roman, et non pas d'une production historiographique. On peut donc se demander si le roman de Cercas met en œuvre une «fabrique de l'histoire» ou une «fabrique de la fiction».

\section{De la fabrique de l'histoire...}

Le personnage principal de Cercas est un journaliste qui s'improvise historien. À travers les pérégrinations intellectuelles de cet apprenti tantôt tenaillé par le doute, tantôt porté par l'espoir, le lecteur assiste à une « fabrique de l'histoire». Une histoire du passé proche qui rend parfois caduques les frontières avec le journalisme et qui s'efforce avec pugnacité de confronter et d'intégrer la mémoire des acteurs survivants à une démarche spécifiquement historique. Le pari est légitime, car si l'histoire se décline en une pluralité de modes elle trouve son unité dans la dimension médiate de la connaissance. C'est à ce prix seul que la fiabilité de la mémoire est interrogée, que le témoignage n'est plus pris pour l'histoire telle quelle mais pour une source préalable à la reconstruction historique. C'est à ce prix seul que l'opération historiogra- 
phique n'est plus un acte de foi mais un acte cognitif prétendant légitimement au statut de science, et que le «devoir d'histoire» ne se laisse pas happer par un «devoir de mémoire» de plus en plus prégnant. À l'insu même des acteurs du drame, le narrateur s'arme donc d'une attitude critique face à une mémoire souvent défaillante.

D'où la multiplication des supports: coupures de presse, archives reproduites, témoignages... D'où le souci constant de traquer l'invraisemblance et le mensonge. Le scripteur accumule les renvois à une réalité extra-textuelle, véritables «marques d'historicité » qui traduisent la volonté de soumettre à un contrôle l'adéquation avec le récit, et qui invitent à reproduire les actes cognitifs, c'est-à-dire le raisonnement ayant permis de trancher entre différentes versions ou de combler les silences de l'histoire. Il montre que l'opération historiographique est d'abord un travail de reconstruction, la mise en série des réponses que l'on tente d'apporter à un questionnement issu d'un certain «lieu d'écriture» et qu'une telle opération est reproductible par n'importe quel lecteur. Ainsi, les deux premiers volets de l'ouvrage de Cercas obéissent aux étapes de l'opération historiographique que distingue Ricoeur $^{2}$ : «Les amis de la forêt » renferment, outre la gestation du récit, la phase documentaire et une partie de la phase explicative/compréhensive; «Les soldats de Salamine » constituent la phase proprement littéraire ou scripturaire qui intègre des éléments de la phase explicative/compréhensive.

Le souci de transparence et l'honnêteté intellectuelle incitent le narrateur à assumer les blancs de l'histoire qu'il n'a pas pu combler. Lorsque les traces de Mazas s'estompent, Cercas se trouve réduit à juxtaposer des témoignages partiels sans pouvoir clarifier la ligne de son personnage. Dans sa mise en scène des doutes de l'historien, il pousse le raffinement jusqu'à les porter à leur paroxysme quand la recherche vacille sous le coup d'un scepticisme généralisé. Au bord du gouffre, le fil du récit est prêt de se rompre car, au royaume du mensonge, l'histoire n'a pas sa place: «Je me dis que, peut-être, toute l'histoire de l'exécution et des circonstances qui l'entouraient pouvait n'être qu'une immense supercherie ${ }^{3}$.» Cercas laisse entendre qu'il n'a pas une prise illimitée sur une réalité qui impose sa loi en dernier

2. Voir Temps et Récit, Paris, Seuil, 1985.

3. Javier Cercas, op. cit., p. 68. 
recours, à la différence du narrateur de roman omnipotent. Le soupçon de travestissement des faits semble ainsi conjuré.

Pour un peu, on en oublierait que Les Soldats de Salamine sont une fiction! Pourtant, Cercas ne s'en cache nullement: l'appartenance au genre romanesque est explicitée sur la couverture de l'ouvrage... Comment alors démêler le vrai du faux de ce complexe écheveau ? Le roman dégage au moins une vérité: une recherche en histoire est d'abord le parcours d'une individualité, avec ses hasards, ses paramètres singuliers qui ont trait à une personne et à une époque, de sorte que l'on retrouve beaucoup de l'historien dans ses écrits, sans qu'il faille y déceler les stigmates d'une quelconque subjectivité.

En emportant son lecteur au point d'éclatement du récit, Cercas, loin d'adopter une démarche d'historien, atteint les sommets de son art de romancier. La mise en question radicale de l'enquête qui menace de rompre la narration est un «effet de réel». Le romancier sur le point d'abandonner sa recherche sur le poète phalangiste veut montrer qu'il n'a affaire qu'au réel, matière incontrôlable qui s'impose à lui. L'illusion du réalisme consiste à déverser le réel dans la fiction, tout en suggérant que, en dernier ressort, c'est le réel qui dicte sa loi au scripteur. C'est la menace même de dissolution du récit qui contribue à renforcer l'illusion romanesque. Cercas feint de pousser la soumission à la sanction du réel au point de renoncer à son projet. Mais l'artifice est bien connu: la progression du fil narratif diffère de l'élaboration du récit par le romancier lui-même. La disproportion entre la réponse au problème (une erreur de transcription) et la remise en cause totale du roman démasque l'artifice: un tel «effet de réel» est un «effet de fiction ». Dans le traitement particulier que fait Cercas d'un réel mis au service de la fiction, les marques d'historicité, ces renvois à un au-delà du texte cautionnant la scientificité de l'opération historiographique, fonctionnent comme un procédé rhétorique pour gagner la confiance du lecteur. Afin de l'emprisonner dans les fils du récit, le romancier imite le chercheur, semblant se conformer à la démarche que Thiers assignait à l'historien : «Être simplement vrai, être ce que sont les choses elles-mêmes, n'être rien de plus qu'elles, n'être rien que par elles, comme elles, autant qu'elles ${ }^{4}$ »

4. Cité par Camille Jullian, Historiens français du XIXe siècle, Paris, Hachette, p. LXIII. 


\section{Les soldats de Salamine}

Cercas ne se contente pas d'un pacte tacite avec son lecteur requérant de ce dernier la suspension volontaire de toute suspicion. Il veut aller plus loin pour prendre l'avantage, et procède à un déplacement fictif des frontières de la fiction. La dynamique textuelle transforme le récit qui devient un piège, de sorte que c'est malgré lui que le lecteur «croit» à la narration. Les Soldats de Salamine répondent alors aux critères du «bon récit» défini par un des personnages, l'écrivain Bolaño : à Cercas qui affirme ne pas entreprendre l'écriture d'un roman mais «une histoire avec des faits et des personnages réels. Un récit réel », il rétorque : "Ça revient au même. Tous les bons récits sont des récits réels, du moins pour celui qui les lit, c'est la seule chose qui compte ${ }^{5}$.» L'ouvrage de Cercas s'approche donc plus d'une réflexion sur la «fabrique» de la fiction que sur la «fabrique» de l'histoire.

\section{... à la fabrique de la fiction}

C'est bien la flamme du romancier qui habite un narrateur devenu journaliste par dépit. Il gravite dans un milieu d'hommes de lettres, et ce sont des entretiens avec des gens de plume qui le mettent sur les traces de Mazas. Enfin, c'est une réflexion d'essence littéraire, la différence entre les héros de caractère et de destin, qui enfante Les Soldats de Salamine.

Son empathie à l'égard du poète phalangiste montre qu'il entretient moins un rapport d'historien que de romancier avec son personnage, et certaines pratiques de Cercas autorisent à voir exclusivement dans Les Soldats de Salamine une fabrique de la fiction; c'est notamment le cas de l'usage du style indirect libre, qui implique la plongée dans le for intérieur de Mazas.

De l'empathie à l'identification et à la fusion, il n'y a qu'un pas que franchit allégrement Cercas. Au milieu du roman, le narrateur «devient» ainsi Mazas, à l'insu du lecteur. Après le récit des principaux faits relatant la jeunesse de cet hidalgo, sa conversion au fascisme, après un glissement révélateur du passé au présent ${ }^{6}$, la froideur du compte rendu s'efface devant une narration chargée d'émotion, où se multiplient notations sensorielles et envolées lyriques à l'approche de

5. Javier Cercas, op. cit., p. 186.

6. P. 97 
l'épisode de la fusillade du Collell. La tension et l'identification de Cercas à Mazas vont crescendo jusqu'à un premier moment d'éternité, celui de la confrontation muette entre le phalangiste et le milicien qui l'épargne. Le récit de cet intense échange de regards où tout se joue est, à quelques détails près, celui de Mazas et non celui de Cercas, qui pourtant tient la plume.

Après avoir tant cédé à la fiction, l'auteur a beau jeu de reprendre le fil du discours de l'historien quand il évoque le sort de Mazas après la guerre civile. Dépouillés de leur chair, les mots retrouvent leur acuité analytique, tandis que le scripteur étale avec ostentation ses scrupules, multiplie les hypothèses triées selon leur vraisemblance. Mais c'est aussi une habileté du romancier que de savoir accorder à la fiction pure toute sa place, pour en recueillir la puissance hallucinatoire tout en masquant son statut d'illusion en l'enchâssant dans un discours historique qui entretient un rapport au réel ontologiquement autre. Ainsi, le deuxième volet du roman est, à quelques agencements près, le récit promis par Mazas aux «amis de la forêt » : «Les soldats de Salamine».

Si les mécanismes de la «fabrique de la fiction», bien qu'affleurant par à-coups, sont dissimulés dans les deux premières parties des Soldats de Salamine, le travail du romancier sur ses personnages est clairement explicité dans le dernier volet consacré à la figure de Miralles. Hantée par la destinée extraordinaire de ce républicain dont Bolaño lui révèle l'existence au hasard d'une conversation, l'imagination du romancier Cercas se met instantanément en branle. Entraîné dans la guerre civile, Miralles passe en France quand Franco triomphe et participe à tous les combats de la Seconde Guerre mondiale, de l'Afrique du Nord à la Normandie en passant par l'Allemagne et l'Autriche, où il marche malencontreusement sur une mine. Son invalidité contraint alors ce héros malgré lui à se retirer à Dijon. Séduit par cette réinsertion d'un anonyme dans la grande geste de l'histoire, Cercas élabore une trame romanesque en une sorte d'écriture mentale.

C'est alors que vient l'illumination, car il ne lui a pas échappé que Miralles fut un soldat de Lister pendant sa retraite en Catalogne à la fin de la guerre civile: «Je m'entendis alors murmurer dans le silence obscur de la chambre: "C'est lui." » Lui, c'est-à-dire le républicain ayant épargné Mazas... Mis au courant des soupçons de son comparse, 


\section{Les soldats de Salamine}

Bolaño lui rétorque qu'il a là un «sacré roman» et que «si ce n'est pas lui, il faut l'inventer ${ }^{7} »$.

Ces quelques lignes montrent comment des éléments réels engendrent et travaillent le récit de fiction. Plusieurs constats en découlent. Il convient de rejeter l'écueil du «fictionnalisme» historique qui, fort du postulat saussurien du langage comme entité close sur elle-même, réduit l'histoire à une branche de la rhétorique, car l'analyse de la frontière mouvante entre histoire et fiction n'a de sens qu'une fois admise une dichotomie dans le rapport au réel de ces deux types d'énoncé. Quoique la fiction se rapproche plus d'une dynamique textuelle que d'une catégorie générique comme l'est le récit historique, elle ne peut se passer du réel de l'histoire qui lui apporte une caution supplémentaire de vérité en masquant la dimension illusoire de la fiction. Mais jusqu'où s'opère la fusion si leurs natures diffèrent?

Pour Bolaño, l'écriture romanesque et la fiction en général sont par essence référentielles. L'écrivain digne de ce nom trouve sa matière brute dans son vécu et sa mémoire: il puise dans ses souvenirs une force impressionniste qui captive le lecteur et lui fait prendre ce qui est dit pour argent comptant. Tout bon récit est donc un récit vrai puisque le lecteur le considère comme tel. On franchit une étape supplémentaire lorsque le référent n'est plus singulier mais universel et historique. La caution de vérité ne réside plus dans la sollicitation du pathos du lecteur, qui s'identifie ou juge de l'authenticité d'un propos en se coulant dans le moule narratif par le truchement de sa mémoire, mais dans les connaissances et la raison du lecteur, car ce référentiel-là se trouve consigné dans d'autres livres ainsi que dans une masse d'archives. Agir sur des réminiscences empreintes d'un sceau de la science qui garantit leur adéquation au réel permet d'authentifier la «vérité» d'un roman: bien employé, un tel déversement de l'histoire dans la fiction constitue un «effet de réel» imparable.

Pourtant, l'adéquation à un vécu personnel ou à un réel «historique » n'est qu' une composante de l'élaboration de la fiction. Et c'est là que se situe le nœud du problème. Car, rappelle Bolaño, si la réalité dépasse parfois la fiction, la fiction résonne parfois plus vrai que la réalité. D'où son conseil à Cercas de renoncer à chercher le vétéran

7. Javier Cercas, op.cit., p. 186. 
catalan dans la mesure où, «inventé, il sera certainement plus réel que le Miralles réel». Il importe avant tout de faire sens car, paradoxalement, le signifiant, même fictif, semble le plus réel. Nul étonnement donc à ce que dans la lettre même, Les Soldats de Salamine plaident pour une hétérogénéité profonde du récit de fiction et du récit historique: leur rapprochement reste asymptotique sans pouvoir opérer de fusion. L'histoire ne se laisse jamais happer par la logique romanesque. Des points de rupture émaillent la diégèse. Pour le lecteur, ces tensions se manifestent par l'intrusion récurrente de marqueurs saturés de signifiance qui viennent construire une atmosphère et parfaire de façon trop évidente un tableau, ou par le sentiment d'être devant le minutieux travail d'un agencement des faits reposant sur la manipulation des temps. Éveillée, la méfiance du lecteur rompt le charme de la fiction. Ce sont les métaphores convenues visant à conférer toute son ampleur morbide au petit matin de l'exécution, ou les jeux temporels qui, brisant la chronologie du récit, détachent des «moments clefs» où tout converge. Quant aux futurs prophétiques ${ }^{8}$, ils sollicitent discrètement le pathos du lecteur en accentuant le tragique de certaines situations et en rendant certains moments exceptionnels par leur signifiance à venir. Ainsi, les histoires de Mazas et de Miralles se trouvent enserrées dans une trame dense qui les transforme en d'authentiques destins.

La quête systématique et la saturation de signifiance finissent par ravaler la première et la dernière partie du roman à un rang aussi illusoire que le récit central dont elles constituaient pourtant la dimension extra-textuelle. Lorsque Cercas, au seuil de son enquête sur Mazas, attend Figueras pour l'interviewer, il entend des gitans jouer Soupir d'Espagne, censé constituer dès cet instant un symbole interne au roman, unifiant tous les niveaux du récit et faisant basculer la réalité référentielle du récit central dans la fiction. Voici annihilé le plus bel «effet de réel» du roman, voici détruit le feuilletage du texte en différentes strates de réalité, indispensable au déplacement fictif des frontières de la fiction au sein d'un unique espace de jeu. La fiction finit

8. Lorsque Mazas en cavale rencontre les «amis de la forêt», le narrateur écrit: «Maria Ferré n'oublierait jamais ce radieux matin de février où elle vit pour la première fois Rafael Sanchez Mazas. » Et de renchérir plus loin: «Soixante ans après, tant Joaquim Figueras que Daniel Angelats se remémoraient encore avec une absolue clarté le matin où ils avaient vu Sanchez Mazas pour la première fois, $[\ldots]$ sa silhouette élancée et aveugle [...] cherchant à tâtons comment sortir du lit pierreux embroussaillé du ruisseau. » 
par se trahir elle-même, victime des lois du genre: l'effet de fiction oscille entre la mise à contribution de l'histoire comme caution de réel, et l'invention créatrice de sens et de signifiance. Deux pôles dont la dimension antithétique tiraille la diégèse jusqu'à en déchirer la surface et laisser visibles les rouages de l'artifice. Mais ce mouvement de balancier qui l'amène à sa perte semble pourtant inévitable car, d'une part, la fiction n'est pas l'histoire et doit s'en séparer par un rapport ontologique différent au réel sous peine de s'y dissoudre et car, d'autre part, parce que la notion matricielle de récit pousse aux glissements de l'une à l'autre. Parce que le récit recourt à un même langage caractérisé par sa transitivité avec le réel, aussi bien pour la fiction que pour l'histoire, il ouvre un éventail de jeux avec le réel pour référentiel. Le récit de fiction peut imiter le récit historique, mais il faut admettre une cassure ontologique et épistémologique radicale entre les deux. L'effet de réel d'une histoire au service de la fiction n'est donc possible que si l'on postule la forme du récit.

On comprend pourquoi l'ouvrage entrepris par Cercas ne pourra finalement pas se nourrir d'un seul corpus documentaire et du croisement de données hétéroclites. C'est que sa démarche est bien celle d'un romancier. Et il finit par admettre: «Si les recherches effectuées sur Mazas constituaient le cœur de mon livre, il arriverait un moment où je devrais me passer de cette béquille, car un écrivain n'écrit jamais sur ce qu'il connaît mais sur ce qu'il ignore.»

\section{Réalité, vérité et signifiance : entre roman «historiographique» et impossible épopée du "présentisme»}

Pourquoi Cercas laisse-t-il sa quête de la signifiance enfermer son propos dans une telle aporie? Certes, l'histoire élabore un effet de réel au service de la fiction; mais le procédé trouve ses limites en se trahissant. Certes, la fiction enrichit l'histoire, joue un rôle heuristique car les prolongements et les variations imaginaires des données de la connaissance engendrent de nouvelles questions, et permet de «faire sentir» l'histoire mieux qu'une froide analyse. Le volet central du roman, «Les soldats de Salamine», ne constitue-t-il pas une des versions des événements portant sur l'exécution manquée du Collell, la mise en action d'une hypothèse raisonnable que rien ne contredit puisque les traces de Sanchez Mazas se perdent complètement? Mais le récit ainsi élaboré n'est pas non plus de l'histoire. Dans ce jeu de 
don et contre-don entre histoire et fiction, les deux y gagnent, jusqu'à s'y perdre...

Peut- être faut-il en revenir à ces soldats de Salamine dont nous n'avons pas dissipé le mystère. Cercas lève le voile en trois temps. Le récit de la confrontation du phalangiste et du républicain pose un jalon. Ce dernier est comparé à un « soldat anonyme et vaincu qui regarde à présent cet homme [Mazas] dont le corps se confond presque avec la terre $^{9} »$ : les frères ennemis incarnent la force déshumanisante de la guerre, quand il n'y a plus de vainqueurs mais des vaincus (un soldat vaincu face à un homme traqué, un anonyme face à un corps boueux retourné à la terre) et quand se perdent les individualités. Cercas insiste sur le motif du double. Ainsi, lorsque Mazas raconte sa confrontation avec le républicain du Collell à Pere, ce «rouge» déserteur, Cercas souligne la relation fusionnelle des compagnons d'infortune. Dos à dos, dans l'obscurité qui empêche de les distinguer, leurs individualités se mêlent pour ne devenir symboliquement que deux cigarettes incandescentes. En outre, Mazas ne cesse de faire le parallèle entre le simple soldat qui l'a épargné et Pere. De sorte que, par ricochet, le républicain du Collell devient le frère et le double positif du poète fasciste. Les propos de Miralles à Cercas apportent un autre éclaircissement: «Ce que vous cherchiez, c'était un héros. Et ce héros, c'était moi ? [...] En temps de paix, il n'y a pas de héros, les véritables héros naissent et meurent dans la guerre. Il n'y a pas de héros vivant. Ils sont morts.» Enfin, dernière pièce du puzzle : "Miralles cessa de parler, sécha ses larmes sans pudeur, comme s'il n'avait pas honte de pleurer en public, $[\ldots]$ ainsi que l'aurait fait un soldat de Salamine ${ }^{10}$.» Reste à opérer la synthèse des indices.

Pour Cercas, il n'y a ni vaincus ni vainqueurs mais des frères ennemis. Ou plutôt, tous sont des vaincus, des soldats de Salamine comme ces Perses laminés, car ceux-là mêmes que le sort des armes a favorisés ne sont plus que des survivants en quittant le champ d'honneur. Tous ont « rendez-vous à Stockton». Stockton, c'est la ville où se déroule le film Fat City, que Bolaño et Miralles avaient vu quand ils fréquentaient le camping de L'Étoile de mer; c'est cette ville où rien ne se passe qui condamne les protagonistes à végéter. L'écrivain chilien raconte l'anec- 
dote à Cercas : "Miralles a dit que nous allions finir de la même manière, comme des ratés, dans une ville atroce, urinant du sang avant de monter sur le ring pour combattre à mort notre propre ombre ${ }^{11}$.» Après l'expérience traumatisante de la guerre, le soldat inconnu finit ses jours à Stockton, invalide, dans une maison de retraite de Dijon. Après l'expérience traumatisante de la guerre, le poète phalangiste se survit dans une existence médiocre aux allures d'anti-destin. Mazas lui aussi finit ses jours à Stockton.

Ce qui explique le conseil donné par Bolaño au narrateur: ne pas chercher à retrouver Miralles, parce qu'inventé il serait plus « réel » que le Miralles vivant. Voilà pourquoi le troisième pan du roman, le récit centré sur la figure de Miralles reste virtuel. Cercas clame l'impossibilité d'un tel récit puisque le Miralles-héros est mort et que seul demeure le Miralles-survivant, sublime et grotesque, vulnérable et éternel. Car avant d'incarner l'hypothétique double républicain de Mazas, Miralles est pour le lecteur ce gros bonhomme au corps bardé de cicatrices qui arbore fièrement ses tongs et son gros ventre au camping de L'Étoile de mer, puis cet homme brisé, difficilement remis d'une embolie cérébrale.

Le versant républicain de la seconde d'éternité de la confrontation des regards avec Mazas ne pourra jamais voir le jour sous la forme d'un récit traditionnel. Cercas ne cherche donc pas à travers l'histoire et la mémoire un effet de réel puisque la rencontre avec le témoin par excellence est un échec. Du coup, les propos désabusés de Cercas, «un romancier n'écrit jamais sur ce qu'il connaît mais sur ce qu'il ignore», prennent une autre ampleur.

Ainsi, lever le mystère sur l'identité des soldats de Salamine pose à nouveau l'énigme de la nature du roman de Cercas. Énigme insoluble? Peut-être pas si l'on considère que le va-et-vient entre plusieurs niveaux de réalité recèle autre chose qu'un déplacement fictif des frontières de la fiction pour piéger le lecteur, et que l'aporie qui tiraillait la diégèse n'en était pas une parce que nous n'avions pas choisi le bon trio en faisant jouer les concepts d'histoire, de fiction et de récit. En cherchant avant tout la signifiance, Cercas ne tente-t-il pas plutôt d'écrire une légende? L'hypothè se séduit, car elle permet d'englober toutes les strates du récit, y compris la réalité extra-textuelle ainsi que

11. Javier Cercas, op. cit., p. 201. 
l'obsession de la mémoire. Les Soldats de Salamine constitueraient une épopée avec un nouveau tragique, où les héros ne finiraient plus sur le champ d'honneur mais se survivraient à Stockton. Une épopée différente de celle qu'aurait écrite Mazas et dont le volet central du roman donne une idée fidèle. Une épopée en rupture avec Eschyle et les écrits grecs évoquant la bataille de Salamine, car l'heure n'est plus aux mythes fondateurs, aux chants des morts triomphalistes qui masquent les fêlures internes.

Que d'écueils pourtant sur la route de Cercas! D'abord, un double paradoxe: on ne peut écrire un récit sans personnages ni héros et pourtant le héros doit rester anonyme; un récit nécessite la succession d'un début, d'un milieu et d'une fin, c'est-à-dire plusieurs instants distincts ; or, pour notre auteur, il n'y a pas de héros mais des attitudes héroïques, le héros ne l'est que l'espace d'un instant. Cercas semble avoir trouvé une parade avec des personnages qui n'ont donc pas de chair, ni de visage, qui sont fils des mots. C'est pourquoi seuls les mots (voix ou écriture) peuvent les dire. D'où la fascination pour le récit de tous les personnages. Mazas est avant tout un homme de lettres qui n'est jamais autant vivant que lorsqu'il raconte et se raconte, ce qu'il ne cesse de faire.

Second apport de Cercas, une construction kaléidoscopique, où l'essentiel du roman tient en quelques «instants de grâce » : un échange de regards au Collell qui trouve une duplication par la force incantatoire du récit lorsque Mazas raconte l'instant d'éternité à Pere ou un paso doble chanté par un soldat républicain enlaçant son fusil, redoublé par le couple improbable d'un vétéran et d'une prostituée dans un camping. Le roman ne pouvait que s'achever sur un récit se niant lui-même, sur le récit virtuel de l'authentique héritier des soldats de Salamine. En outre, cet agencement des faits évoque plus que le récit, la notion de «mise en intrigue», qui permet aussi bien d'osciller entre histoire et fiction tout en préservant leur spécificité.

Grâce à cette esthétique du collage, Cercas permet la confrontation d'une nation n'ayant pas entrepris son travail de mémoire avec le passé douloureux de la guerre civile. Le roman apparaît à un moment particulier de l'histoire espagnole, après la phase d'occultation de la mémoire nécessaire à l'apaisement souhaité par la transition démocratique, après la phase de désinhibition de certains franquistes, au moment où le futur semble incertain et le passé déchiré. Si l'on reprend 


\section{Les soldats de Salamine}

la terminologie de Hartog ${ }^{12}$, Les Soldats de Salamine offrent la radiographie d'un certain régime d'historicité, c'est-à-dire d'une articulation particulière du présent du passé et du futur que l'on pourrait qualifier de «présentisme». En incarnant l'impossibilité d'une légende, l'ouvrage témoigne de cette invasion par le présent, du passé et du futur, parce que le passé n'offre plus un socle assez solide pour porter le présent, et que le futur ne porte plus d'espoir pour l'inspirer. La quête d'une mémoire libératrice sous-jacente aux Soldats de Salamine imposait le genre de l'épopée, mais nouvelle manière. Cercas témoigne de ce que le présent doit assumer son passé par un dosage de mémoire, d'histoire et d'oubli pour permettre la construction d'un futur libéré de ses séquelles et aller «de l'avant, de l'avant, toujours de l'avant» (aphorisme qui clôt le roman), sans haine, ni honte. Ainsi, on passe du trio histoire, fiction et récit à celui d'épopée, régime d'historicité et mise en intrigue. Quant à l'historien, de façon moins douloureuse, il peut y voir un roman historiographique où se mêlent épopée et travail minutieux de mise en intrigue au service d'un «présentisme».

12. Régimes d'historicité. Présentisme et expérience du temps, Paris, Seuil, 2003. 